\title{
The noncovariant gauges in 3-form theories
}

\author{
Sudhaker Upadhyay*, Manoj Kumar Dwivedi ${ }^{\dagger}$, and Bhabani Prasad Mandal ${ }^{\ddagger}$ \\ Department of Physics, Banaras Hindu University, Varanasi-221005, INDIA.
}

\begin{abstract}
We study the 3-form gauge theory in the context of generalized BRST formulation. We construct the finite field-dependent BRST (FFBRST) symmetry for such a theory. The generating functional for 3 -form gauge theory in noncovariant gauge is obtained from that of in covariant gauge. We further extend the results by considering 3-form gauge theory in the context of Batalin-Vilkovisky (BV) formulation.
\end{abstract}

\section{INTRODUCTION}

Calculations in the gauge field theory have been done in variety of gauges depending on the case and convenience of the calculation. The gauge theories in noncovariant gauges have been a subject of wide research due to certain advantages in these gauges [1-6]. In particular, the confinement problem of QCD in Coulomb gauge [4], superstring theory in light-cone gauge [5] are more tractable and the ultraviolet finiteness of supersymmetric YangMills theory are more transparent in noncovariant gauges [6] .

In this present work we would like to show how noncovariant gauge formulation of Abelian 3-form gauge theory can be developed by making a connection of the same theory in covariant gauge. A 3 -form field theory is subject of interest as a 3form $\mathrm{C}$ field arises naturally in M-theory. The theory of multiple M2-branes has been used to study M5-branes and a 3-form field naturally occurs in this theory [7, 8]. By considering a system of M2-branes ending on an M5-brane with a constant 3-form field turned on, the Bagger-LambertGustavsson (BLG) model was used to motivate a novel quantum geometry on the M5-brane worldvolume [9]. In particular, the action for multiple M2-branes was studied via BLG theory [10-13]. The BRST of BLG theory has been studied recently [14].

Another interesting relation between multiple M2-branes and the M5-brane is the identification of the BLG action (with Nambu-Poisson 3-bracket) as the M5-brane action with a large world volume 3 -form field [15]. The quantization of the higher form gauge theories is also very important as these

\footnotetext{
*e-mail address: sudhakerupadhyay@gmail.com

†e-mail address: manojdwivedi84@gmail.com

${ }^{\ddagger}$ e-mail address: bhabani.mandal@gmail.com
}

fields play the important roles in the excitations of the quantized versions of strings, superstrings and other extended objects [16 18]. Recently, the BRST and BV quantizations of Abelian 3-form gauge theory in covariant gauge have been studied extensively [19].

We show in this work how the generating functional of 3 -form gauge theory in covariant gauge is related to that of in the noncovariant gauges by using FFBRST transformation. The FFBRST transformation of this theory is constructed by making the infinitesimal parameter finite and fielddependent. The FFBRST transformations are very useful in connecting different effective theories and hence found many applications [20 28]. Such a generalized BRST transformation has also been studied in the context of BV formulation [26, 29]. We consider axial gauge in this work as an example of noncovariant gauge. However, our results are general and are valid for any noncovariant gauge. We further show the mapping between the generating functionals of 3-form gauge theories in covariant and noncovariant gauge in $\mathrm{BV}$ formulation also.

The plan of the paper is as follows. We start with a brief discussion of Abelian 3-form gauge theory in Sec. II. The section III is devoted to generalization of BRST transformation. In Sec. IV, we show the connection between the Abelian 3form gauge theories in covariant and noncovariant gauges. The BV formulation for such a theory is discussed in section $\mathrm{V}$. The last section is reserved for concluding remarks.

\section{ABELIAN 3-FORM GAUGE THEORIES}

We start with the classical action for the Abelian 3 -form gauge theory [19] in $(1+5)$ dimensions as

$$
S_{0}=\frac{1}{24} \int d^{6} x H_{\mu \nu \eta \chi} H^{\mu \nu \eta \chi},
$$


where the field strength (curvature) tensor in terms of totally antisymmetric tensor gauge field $B_{\mu \nu \eta}$ is defined as

$$
H_{\mu \nu \eta \chi}=\partial_{\mu} B_{\nu \eta \chi}-\partial_{\nu} B_{\eta \chi \mu}+\partial_{\eta} B_{\chi \mu \nu}-\partial_{\xi} B_{\mu \nu \eta} .
$$

This Lagrangian density is invariant under the infinitesimal gauge transformation defined as

$$
\delta B_{\mu \nu \eta}=\partial_{\mu} \lambda_{\nu \eta}+\partial_{\nu} \lambda_{\eta \mu}+\partial_{\eta} \lambda_{\mu \nu},
$$

where $\lambda_{\mu \nu}$ is an arbitrary antisymmetric parameter. To incorporate the BRST symmetry in this system, we extend the action by introducing the following covariant gauge-fixing and ghost terms as [19]:

$$
\begin{aligned}
S_{g f+g h} & =\int d^{6} x\left[\partial_{\mu} B^{\mu \nu \eta} B_{\nu \eta}+\frac{1}{2} B_{\mu \nu} \tilde{B}^{\mu \nu}\right. \\
& +\left(\partial_{\mu} \tilde{c}_{\nu \eta}+\partial_{\nu} \tilde{c}_{\eta \mu}+\partial_{\eta} \tilde{c}_{\mu \nu}\right) \partial^{\mu} c^{\nu \eta} \\
& -\left(\partial_{\mu} \tilde{\beta}_{\nu}-\partial_{\nu} \tilde{\beta}_{\mu}\right) \partial^{\mu} \beta^{\nu}-B B_{2} \\
& -\frac{1}{2} B_{1}^{2}+\left(\partial_{\mu} \tilde{c}^{\mu \nu}\right) f_{\nu}-\left(\partial_{\mu} c^{\mu \nu}\right) \tilde{F}_{\nu} \\
& +\partial_{\mu} \tilde{c}_{2} \partial^{\mu} c_{2}+\tilde{f}_{\mu} f^{\mu}-\tilde{F}_{\mu} F^{\mu} \\
& \left.+\partial_{\mu} \beta^{\mu} B_{2}+\partial_{\mu} \phi^{\mu} B_{1}-\partial_{\mu} \tilde{\beta}^{\mu} B\right]
\end{aligned}
$$

where antisymmetric ghost field $c_{\mu \nu}$ and antisymmetric antighost field $\tilde{c}_{\mu \nu}$ are fermionic in nature and the vector field $\phi_{\mu}$, antisymmetric auxiliary fields $B_{\mu \nu}, \tilde{B}_{\mu \nu}$ and auxiliary fields $B, B_{1}, B_{2}$ are bosonic in nature. Rest of the fields $f_{\mu}, \tilde{f}_{\mu}, F_{\mu}$ and $\tilde{F}_{\mu}$ are auxiliary Grassmannian fields.

The complete effective action for Abelian 3-form gauge theory is then written as

$$
S_{e f f}=S_{0}+S_{g f+g h} .
$$

This effective action $\left(S_{e f f}\right)$ is invariant under following BRST transformation:

$$
\begin{aligned}
\delta_{b} B_{\mu \nu \eta} & =-\left(\partial_{\mu} c_{\nu \eta}+\partial_{\nu} c_{\eta \mu}+\partial_{\eta} c_{\mu \nu}\right) \delta \Lambda \\
\delta_{b} c_{\mu \nu} & =\left(\partial_{\mu} \beta_{\nu}-\partial_{\nu} \beta_{\mu}\right) \delta \Lambda, \quad \delta_{b} \tilde{c}_{\mu \nu}=B_{\mu \nu} \delta \Lambda \\
\delta_{b} \tilde{B}_{\mu \nu} & =-\left(\partial_{\mu} f_{\nu}-\partial_{\nu} f_{\mu}\right) \delta \Lambda, \quad \delta_{b} \tilde{\beta}_{\mu}=-\tilde{F}_{\mu} \delta \Lambda \\
\delta_{b} \beta_{\mu} & =-\partial_{\mu} c_{2} \delta \Lambda, \quad \delta_{b} F_{\mu}=-\partial_{\mu} B \delta \Lambda \\
\delta_{b} \tilde{c}_{2} & =B_{2} \delta \Lambda, \quad \delta_{b} \tilde{f}_{\mu}=\partial_{\mu} B_{1} \delta \Lambda \\
\delta_{b} c_{1} & =-B \delta \Lambda, \quad \delta_{b} \phi_{\mu}=-f_{\mu} \delta \Lambda \\
\delta_{b} \tilde{c}_{1} & =B_{1} \delta \Lambda, \quad \delta_{b} \mathcal{M}=0 \\
\mathcal{M} & \equiv\left\{c_{2}, f_{\mu}, \tilde{F}_{\mu}, B, B_{1}, B_{2}, B_{\mu \nu}\right\}
\end{aligned}
$$

where $\delta \Lambda$ is infinitesimal, anticommuting and global parameter. The gauge-fixing and ghost part of the effective action, $S_{g f+g h}$, is separately BRST invariant and is written in terms of BRST variation of the gauge-fixed fermion $\left(\Psi_{L}\right)$ as

$$
\begin{aligned}
S_{g f+g h} & =s_{b} \Psi_{L}=s_{b} \int d^{6} x\left[-\partial_{\mu} \tilde{c}_{\nu \eta} B^{\mu \nu \eta}-\frac{1}{2} \tilde{c}_{2} B\right. \\
& +\frac{1}{2} c_{1} B_{2}-\frac{1}{2} \tilde{c}_{1} B_{1}-c^{\mu \nu} \partial_{\mu} \tilde{\beta}_{\nu}-\partial_{\mu} \tilde{c}_{2} \beta^{\mu} \\
& \left.+\frac{1}{2} \tilde{c}_{\mu \nu} \tilde{B}^{\mu \nu}-F^{\mu} \tilde{\beta}_{\mu}-\tilde{f}^{\mu} \phi_{\mu}\right],
\end{aligned}
$$

where the expression for $\Psi_{L}$ is given as

$$
\begin{aligned}
\Psi_{L} & =\int d^{6} x \psi_{L}=\int d^{6} x\left[-\partial_{\mu} \tilde{c}_{\nu \eta} B^{\mu \nu \eta}-\frac{1}{2} \tilde{c}_{2} B\right. \\
& +\frac{1}{2} c_{1} B_{2}-\frac{1}{2} \tilde{c}_{1} B_{1}-c^{\mu \nu} \partial_{\mu} \tilde{\beta}_{\nu}-\partial_{\mu} \tilde{c}_{2} \beta^{\mu} \\
& \left.+\frac{1}{2} \tilde{c}_{\mu \nu} \tilde{B}^{\mu \nu}-F^{\mu} \tilde{\beta}_{\mu}-\tilde{f}^{\mu} \phi_{\mu}\right] .
\end{aligned}
$$

The generating functional for such a theory is defined in the path integral formulation as,

$$
Z_{\text {eff }}=\int \mathcal{D} \phi e^{i S_{\text {eff }}},
$$

where $\mathcal{D} \phi$ is the path integral measure which includes all the fields $\phi$, generically.

\section{GENERALIZED BRST FORMULATION OF ABELIAN 3-FORM GAUGE THEORY}

The properties of the usual BRST transformation in Eq. (6) do not depend on whether the parameter $\delta \Lambda$ is (i) finite or infinitesimal, (ii) fielddependent or not, as long as it is anticommuting and space-time independent. These observations give us a freedom to generalize the BRST transformation by making the parameter, $\delta \Lambda$ finite and field-dependent without affecting its properties. Such generalized BRST transformation is known as finite field-dependent BRST (FFBRST) transformation [20]. To generalize the BRST transformation we start by making the infinitesimal parameter field-dependent with introduction of an arbitrary parameter $\kappa(0 \leq \kappa \leq 1)$. We allow the fields, $\phi(x, \kappa)$, to depend on $\kappa$ in such a way that $\phi(x, \kappa=0)=\phi(x)$ and $\phi(x, \kappa=1)=\phi^{\prime}(x)$, the transformed field.

The usual infinitesimal BRST transformation, thus can be written generically as

$$
d \phi(x, \kappa)=s_{b}[\phi(x, \kappa)] \Theta^{\prime}[\phi(x, \kappa)] d \kappa
$$


where the $\Theta^{\prime}[\phi(x, \kappa)] d \kappa$ is the infinitesimal but field-dependent parameter. The FFBRST transformation with the finite field-dependent parameter then can be constructed by integrating such infinitesimal transformation from $\kappa=0$ to $\kappa=1$, to obtain

$$
\phi^{\prime} \equiv \phi(x, \kappa=1)=\phi(x, \kappa=0)+s_{b}[\phi(x)] \Theta[\phi(x)]
$$

where

$$
\Theta[\phi(x)]=\int_{0}^{1} d \kappa^{\prime} \Theta^{\prime}\left[\phi\left(x, \kappa^{\prime}\right)\right],
$$

is the finite field-dependent parameter [20].

The FFBRST transformation is constructed in this case as follows:

$$
\begin{aligned}
& \delta_{b} B_{\mu \nu \eta}=-\left(\partial_{\mu} c_{\nu \eta}+\partial_{\nu} c_{\eta \mu}+\partial_{\eta} c_{\mu \nu}\right) \Theta[\phi], \\
& \delta_{b} c_{\mu \nu}=\left(\partial_{\mu} \beta_{\nu}-\partial_{\nu} \beta_{\mu}\right) \Theta[\phi], \\
& \delta_{b} \tilde{c}_{\mu \nu}=B_{\mu \nu} \Theta[\phi], \quad \delta_{b} \tilde{\beta}_{\mu}=-\tilde{F}_{\mu} \Theta[\phi], \\
& \delta_{b} \tilde{B}_{\mu \nu}=-\left(\partial_{\mu} f_{\nu}-\partial_{\nu} f_{\mu}\right) \Theta[\phi], \\
& \delta_{b} \beta_{\mu}=-\partial_{\mu} c_{2} \Theta[\phi], \quad \delta_{b} F_{\mu}=-\partial_{\mu} B \Theta[\phi], \\
& \delta_{b} \tilde{c}_{2}=B_{2} \Theta[\phi], \quad \delta_{b} \tilde{f}_{\mu}=\partial_{\mu} B_{1} \Theta[\phi], \\
& \delta_{b} c_{1}=-B \Theta[\phi], \quad \delta_{b} \phi_{\mu}=-f_{\mu} \Theta[\phi], \\
& \delta_{b} \tilde{c}_{1}=B_{1} \Theta[\phi], \quad \delta_{b} \varpi=0, \\
& \varpi \equiv\left[c_{2}, f_{\mu}, \tilde{F}_{\mu}, B, B_{1}, B_{2}, B_{\mu \nu}\right],
\end{aligned}
$$

Such an off-shell nilpotent BRST transformation with finite field-dependent parameter is the symmetry of the effective action in Eq. (5). However, the path integral measure in Eq. (9) is not invariant under such transformation as the BRST parameter is finite in nature.

The Jacobian of the path integral measure for such transformations is then evaluated for some particular choices of the finite field-dependent parameter, $\Theta[\phi(x)]$, as

$$
\mathcal{D} \phi^{\prime}=J(\kappa) \mathcal{D} \phi(\kappa) .
$$

The Jacobian, $J(\kappa)$ can be replaced (within the functional integral) as

$$
J(\kappa) \rightarrow \exp \left[i S_{1}[\phi(x, \kappa)]\right]
$$

iff the following condition is satisfied [20]

$$
\begin{aligned}
& \int \mathcal{D} \phi(x)\left[\frac{1}{J} \frac{d J}{d \kappa}-i \frac{d S_{1}[\phi(x, \kappa)]}{d \kappa}\right] \times \\
& \exp \left[i\left(S_{\text {eff }}+S_{1}\right)\right]=0
\end{aligned}
$$

where $S_{1}[\phi]$ is local functional of fields.
The infinitesimal change in the $J(\kappa)$ is written as

$$
\frac{1}{J} \frac{d J}{d \kappa}=-\int d^{6} y\left[ \pm \delta_{b} \phi(y, \kappa) \frac{\partial \Theta^{\prime}[\phi(y, \kappa)]}{\partial \phi(y, \kappa)}\right],
$$

where \pm sign refers to whether $\phi$ is a bosonic or a fermionic field.

By constructing appropriate $\Theta$, we can change $S_{1}$ in such a manner that $S_{\text {eff }}+S_{1}$ becomes a new effective action.

\section{3-FORM GAUGE THEORY IN NONCOVARIANT GAUGE}

To obtain the generating functional for 3 -form gauge theory in noncovariant gauge we construct the infinitesimal field-dependent parameter as

$$
\begin{aligned}
\Theta^{\prime} & =i \gamma \int d^{6} y\left[-\tilde{c}_{\nu \eta} \partial_{\mu} B^{\mu \nu \eta}+\tilde{c}_{\nu \eta} \eta_{\mu} B^{\mu \nu \eta}\right. \\
& +c_{\mu \nu} \partial^{\mu} \tilde{\beta}^{\nu}-c_{\mu \nu} \eta^{\mu} \tilde{\beta}^{\nu}-\tilde{c}_{2} \partial_{\mu} \beta^{\mu} \\
& \left.+\tilde{c}_{2} \eta_{\mu} \beta^{\mu}\right],
\end{aligned}
$$

where $\gamma$ is an arbitrary constant parameter.

Using Eq. (17), we calculate the infinitesimal change in Jacobian of functional integral as

$$
\begin{aligned}
\frac{1}{J} \frac{d J}{d \kappa} & =-i \gamma \int d^{6} y\left[B_{\nu \eta} \partial_{\mu} B^{\mu \nu \eta}-B_{\nu \eta} \eta_{\mu} B^{\mu \nu \eta}\right. \\
& +\partial^{\mu}\left(\partial_{\mu} c_{\nu \eta}+\partial_{\nu} c_{\eta \mu}+\partial_{\eta} c_{\mu \nu}\right) \tilde{c}^{\nu \eta} \\
& -\eta^{\mu}\left(\partial_{\mu} c_{\nu \eta}+\partial_{\nu} c_{\eta \mu}+\partial_{\eta} c_{\mu \nu}\right) \tilde{c}^{\nu \eta} \\
& -\left(\partial_{\mu} \tilde{\beta}_{\nu}-\partial_{\nu} \tilde{\beta}_{\mu}\right) \partial^{\mu} \beta^{\nu}+c_{\mu \nu} \partial^{\mu} \tilde{F}^{\nu} \\
& +\left(\eta_{\mu} \tilde{\beta}_{\nu}-\eta_{\nu} \tilde{\beta}_{\mu}\right) \partial^{\mu} \beta^{\nu}-c_{\mu \nu} \eta^{\mu} \tilde{F}^{\nu} \\
& +B_{2} \partial_{\mu} \beta^{\mu}-B_{2} \eta_{\mu} \beta^{\mu}-\tilde{c}_{2} \partial_{\mu} \partial^{\mu} c_{2} \\
& \left.+\tilde{c}_{2} \eta_{\mu} \partial^{\mu} c_{2}\right] .
\end{aligned}
$$

The Jacobian $J$ can be written as $e^{i S_{1}}$ when the condition (16) is satisfied. We make the following ansatz for functional $S_{1}$ in this case:

$$
\begin{aligned}
S_{1} & =\int d^{6} x\left[\xi_{1}(\kappa) B_{\nu \eta} \partial_{\mu} B^{\mu \nu \eta}+\xi_{2}(\kappa) B_{\nu \eta} \eta_{\mu} B^{\mu \nu \eta}\right. \\
& +\xi_{3}(\kappa) \partial^{\mu}\left(\partial_{\mu} c_{\nu \eta}+\partial_{\nu} c_{\eta \mu}+\partial_{\eta} c_{\mu \nu}\right) \tilde{c}^{\nu \eta} \\
& +\xi_{4}(\kappa) \eta^{\mu}\left(\partial_{\mu} c_{\nu \eta}+\partial_{\nu} c_{\eta \mu}+\partial_{\eta} c_{\mu \nu}\right) \tilde{c}^{\nu \eta} \\
& +\xi_{5}(\kappa)\left(\partial_{\mu} \tilde{\beta}_{\nu}-\partial_{\nu} \tilde{\beta}_{\mu}\right) \partial^{\mu} \beta^{\nu} \\
& +\xi_{6}(\kappa)\left(\eta_{\mu} \tilde{\beta}_{\nu}-\eta_{\nu} \tilde{\beta}_{\mu}\right) \partial^{\mu} \beta^{\nu} \\
& +\xi_{7}(\kappa) c_{\mu \nu} \partial^{\mu} \tilde{F}^{\nu}+\xi_{8}(\kappa) c_{\mu \nu} \eta^{\mu} \tilde{F}^{\nu} \\
& +\xi_{9}(\kappa) B_{2} \partial_{\mu} \beta^{\mu}+\xi_{10}(\kappa) B_{2} \eta_{\mu} \beta^{\mu} \\
& \left.+\xi_{11}(\kappa) \tilde{c}_{2} \partial_{\mu} \partial^{\mu} c_{2}+\xi_{12}(\kappa) \tilde{c}_{2} \eta_{\mu} \partial^{\mu} c_{2}\right] . \quad(20)
\end{aligned}
$$


Now, the condition (16) yields

$$
\begin{aligned}
& \int \mathcal{D} \phi(x)\left[\left(\xi_{1}^{\prime}+\gamma\right) B_{\nu \eta} \partial_{\mu} B^{\mu \nu \eta}\right. \\
+ & \left(\xi_{2}^{\prime}-\gamma\right) B_{\nu \eta} \eta_{\mu} B^{\mu \nu \eta} \\
+ & \left(\xi_{3}^{\prime}+\gamma\right) \partial^{\mu}\left(\partial_{\mu} c_{\nu \eta}+\partial_{\nu} c_{\eta \mu}+\partial_{\eta} c_{\mu \nu}\right) \tilde{c}^{\nu \eta} \\
+ & \left(\xi_{4}^{\prime}-\gamma\right) \eta^{\mu}\left(\partial_{\mu} c_{\nu \eta}+\partial_{\nu} c_{\eta \mu}+\partial_{\eta} c_{\mu \nu}\right) \tilde{c}^{\nu \eta} \\
+ & \left(\xi_{5}^{\prime}-\gamma\right)\left(\partial_{\mu} \tilde{\beta}_{\nu}-\partial_{\nu} \tilde{\beta}_{\mu}\right) \partial^{\mu} \beta^{\nu} \\
+ & \left(\xi_{6}^{\prime}+\gamma\right)\left(\eta_{\mu} \tilde{\beta}_{\nu}-\eta_{\nu} \tilde{\beta}_{\mu}\right) \partial^{\mu} \beta^{\nu} \\
+ & \left(\xi_{7}^{\prime}+\gamma\right) c_{\mu \nu} \partial^{\mu} \tilde{F}^{\nu}+\left(\xi_{8}^{\prime}-\gamma\right) c_{\mu \nu} \eta^{\mu} \tilde{F}^{\nu} \\
+ & \left(\xi_{9}^{\prime}+\gamma\right) B_{2} \partial_{\mu} \beta^{\mu}+\left(\xi_{10}^{\prime}-\gamma\right) B_{2} \eta_{\mu} \beta^{\mu} \\
+ & \left(\xi_{11}^{\prime}-\gamma\right) \tilde{c}_{2} \partial_{\mu} \partial^{\mu} c_{2}+\left(\xi_{12}^{\prime}+\gamma\right) \tilde{c}_{2} \eta_{\mu} \partial^{\mu} c_{2} \\
- & \beta_{\nu \eta} \partial_{\mu}\left(\partial^{\mu} c^{\nu \eta}+\partial^{\nu} c^{\eta \mu}+\partial^{\eta} c^{\mu \nu}\right) \Theta^{\prime}\left(\xi_{1}-\xi_{3}\right) \\
- & \beta_{\nu \eta} \eta_{\mu}\left(\partial^{\mu} c^{\nu \eta}+\partial^{\nu} c^{\eta \mu}+\partial^{\eta} c^{\mu \nu}\right) \Theta^{\prime}\left(\xi_{2}-\xi_{4}\right) \\
- & \left(\partial_{\mu} \tilde{F}_{\nu}-\partial_{\nu} \tilde{F}_{\mu}\right) \partial^{\mu} \beta^{\nu} \Theta^{\prime}\left(\xi_{5}+\xi_{7}\right) \\
- & \left(\eta_{\mu} \tilde{F}_{\nu}-\eta_{\nu} \tilde{F}_{\mu}\right) \partial^{\mu} \beta^{\nu} \Theta^{\prime}\left(\xi_{6}+\xi_{8}\right) \\
- & B_{2} \partial_{\mu} \partial^{\mu} c_{2} \Theta^{\prime}\left(\xi_{9}+\xi_{11}\right) \\
- & \left.B_{2} \eta_{\mu} \partial^{\mu} c_{2} \Theta^{\prime}\left(\xi_{10}+\xi_{12}\right)\right]=0 .
\end{aligned}
$$

The nonlocal ( $\Theta^{\prime}$ dependent $)$ terms will vanish if the following conditions are satisfied:

$$
\begin{aligned}
& \xi_{1}-\xi_{3}=\xi_{2}-\xi_{4}=\xi_{5}+\xi_{7}= \\
& \xi_{6}+\xi_{8}=\xi_{9}+\xi_{11}=\xi_{10}+\xi_{12}=0 .
\end{aligned}
$$

Equating the L.H.S. and R.H.S. of the Eq. (21) we get the following differential equations:

$$
\begin{aligned}
\xi_{1}^{\prime}+\gamma=0, & \xi_{2}^{\prime}-\gamma=0, \\
\xi_{3}^{\prime}+\gamma=0, & \xi_{4}^{\prime}-\gamma=0, \\
\xi_{5}^{\prime}-\gamma=0, & \xi_{6}^{\prime}+\gamma=0, \\
\xi_{7}^{\prime}+\gamma=0, & \xi_{8}^{\prime}-\gamma=0, \\
\xi_{9}^{\prime}+\gamma=0, & \xi_{10}^{\prime}-\gamma=0, \\
\xi_{11}^{\prime}-\gamma=0, & \xi_{12}^{\prime}+\gamma=0 .
\end{aligned}
$$

The solutions of the above differential equations satisfying the initial conditions, i.e. $\xi_{i}(\kappa=0)=$ $0(i=1,2, \ldots, 12)$, are

$$
\begin{aligned}
\xi_{1}=-\gamma \kappa, \quad \xi_{2}=\gamma \kappa, & \xi_{3}=-\gamma \kappa, \\
\xi_{4}=\gamma \kappa, \quad \xi_{5}=\gamma \kappa, & \xi_{6}=-\gamma \kappa, \\
\xi_{7}=-\gamma \kappa, \quad \xi_{8}=\gamma \kappa, & \xi_{9}=-\gamma \kappa, \\
\xi_{10}=\gamma \kappa, \quad \xi_{11}=\gamma \kappa, & \xi_{12}=-\gamma \kappa .
\end{aligned}
$$

Plugging back these values, the expression of $S_{1}$ given in Eq. (20) becomes

$$
S_{1}=\int d^{6} x\left[-\gamma \kappa B_{\nu \eta} \partial_{\mu} B^{\mu \nu \eta}+\gamma \kappa B_{\nu \eta} \eta_{\mu} B^{\mu \nu \eta}\right.
$$

$$
\begin{aligned}
& -\gamma \kappa \partial^{\mu}\left(\partial_{\mu} c_{\nu \eta}+\partial_{\nu} c_{\eta \mu}+\partial_{\eta} c_{\mu \nu}\right) \tilde{c}^{\nu \eta} \\
& +\gamma \kappa \eta^{\mu}\left(\partial_{\mu} c_{\nu \eta}+\partial_{\nu} c_{\eta \mu}+\partial_{\eta} c_{\mu \nu}\right) \tilde{c}^{\nu \eta} \\
& +\gamma \kappa\left(\partial_{\mu} \tilde{\beta}_{\nu}-\partial_{\nu} \tilde{\beta}_{\mu}\right) \partial^{\mu} \beta^{\nu} \\
& -\gamma \kappa\left(\eta_{\mu} \tilde{\beta}_{\nu}-\eta_{\nu} \tilde{\beta}_{\mu}\right) \partial^{\mu} \beta^{\nu} \\
& -\gamma \kappa c_{\mu \nu} \partial^{\mu} \tilde{F}^{\nu}+\gamma \kappa c_{\mu \nu} \eta^{\mu} \tilde{F}^{\nu} \\
& -\gamma \kappa B_{2} \partial_{\mu} \beta^{\mu}+\gamma \kappa B_{2} \eta_{\mu} \beta^{\mu} \\
& \left.+\gamma \kappa \tilde{c}_{2} \partial_{\mu} \partial^{\mu} c_{2}-\gamma \kappa \tilde{c}_{2} \eta_{\mu} \partial^{\mu} c_{2}\right] .
\end{aligned}
$$

We consider the arbitrary constant $\gamma=1$ in the above expression without any loss of generality and by adding the expression (25) at $\kappa=1$ to the effective action for 3 -form gauge theory in covariant gauge, we get

$$
\begin{aligned}
S_{e f f} & +S_{1}(\kappa=1)=\int d^{6} x\left[\frac{1}{24} H_{\mu \nu \eta \chi} H^{\mu \nu \eta \chi}\right. \\
& +\eta_{\mu} B^{\mu \nu \eta} B_{\nu \eta}+\frac{1}{2} B_{\mu \nu} \tilde{B}^{\mu \nu} \\
& +\left(\partial_{\mu} \tilde{c}_{\nu \eta}+\partial_{\nu} \tilde{c}_{\eta \mu}+\partial_{\eta} \tilde{c}_{\mu \nu}\right) \eta^{\mu} c^{\nu \eta} \\
& -\left(\eta_{\mu} \tilde{\beta}_{\nu}-\eta_{\nu} \tilde{\beta}_{\mu}\right) \partial^{\mu} \beta^{\nu}-B B_{2} \\
& -\frac{1}{2} B_{1}^{2}+\left(\partial_{\mu} \tilde{c}^{\mu \nu}\right) f_{\nu}+c_{\mu \nu} \eta^{\mu} \tilde{F}^{\nu} \\
& -\tilde{c}_{2} \eta_{\mu} \partial^{\mu} c_{2}+\tilde{f}_{\mu} f^{\mu}-\tilde{F}_{\mu} F^{\mu} \\
& \left.+\eta_{\mu} \beta^{\mu} B_{2}+\partial_{\mu} \phi^{\mu} B_{1}-\partial_{\mu} \tilde{\beta}^{\mu} B\right] .
\end{aligned}
$$

This is nothing but the 3 -form effective action in noncovariant gauge.

We end this this section by making the comment that the noncovariant gauge formulation of 3 -form gauge theory is obtained from covariant gauge formulation of the same through a FFBRST transformation with appropriate finite field dependent parameter. Even though we have established the connection between generating functionals in Lorentz (covariant) gauge and in axial (noncovariant) gauge our formulation is valid for the connection between generating functionals in any covariant and in any noncovariant gauges.

\section{MAPPING OF COVARIANT AND NONCOVARIANT GAUGES IN 3-FORM GAUGE THEORY: BV FORMULATION}

In this section, we consider the BV formulation for Abelian 3-form gauge theory to reestablish the results of the previous section. For this purpose we express the generating functional in Eq. (9) in field/antifield formulation by introducing the antifield $\phi^{\star}$ corresponding to each generic field $\phi$ with 
opposite statistics, as

$$
\begin{aligned}
Z_{e f f} & =\int \mathcal{D} \phi \exp \left[i \int d ^ { 6 } x \left\{\frac{1}{24} F_{\mu \nu \eta \chi} F^{\mu \nu \eta \chi}\right.\right. \\
& -B_{\mu \nu \eta}^{\star}\left(\partial^{\mu} c^{\nu \eta}+\partial^{\nu} c^{\eta \mu}+\partial^{\eta} c^{\mu \nu}\right) \\
& +c_{\mu \nu}^{\star}\left(\partial^{\mu} \beta^{\nu}-\partial^{\nu} \beta^{\mu}\right)+\tilde{c}_{\mu \nu}^{\star} B^{\mu \nu} \\
& -\tilde{B}_{\mu \nu}^{\star}\left(\partial^{\mu} f^{\nu}-\partial^{\nu} f^{\mu}\right)-\beta_{\mu}^{\star} \partial^{\mu} c_{2}-\tilde{\beta}_{\mu}^{\star} \tilde{F}^{\mu} \\
& -F_{\mu}^{\star} \partial^{\mu} B+\tilde{f}_{\mu}^{\star} \partial^{\mu} B_{1}+\tilde{c}_{2}^{\star} B_{2}+\tilde{c}_{1}^{\star} B_{1} \\
& \left.\left.-c_{1}^{\star} B-\phi_{\mu}^{\star} f^{\mu}\right\}\right] .
\end{aligned}
$$

This further can be written in compact form as

$$
Z_{e f f}=\int \mathcal{D} \phi e^{i W_{\Psi_{L}}\left(\phi, \phi^{\star}\right)},
$$

where $W_{\Psi_{L}}\left(\phi, \phi^{\star}\right)$ is an extended action for Abelian 3-form gauge theory in the covariant gauge corresponding to the gauge-fixed fermion $\Psi_{L}$ (Eq. (8)) having Grassmann parity 1 and ghost number -1 .

The generating functional $Z_{\text {eff }}$ does not depend on the choice of gauge-fixed fermion [30]. This extended quantum action, $W_{\Psi_{L}}\left(\phi, \phi^{\star}\right)$, satisfies certain rich mathematical relation which is called quantum master equation [31] and is given by

$$
\Delta e^{i W_{\Psi_{L}}\left[\phi, \phi^{\star}\right]}=0, \quad \Delta \equiv \frac{\partial_{r}}{\partial \phi} \frac{\partial_{r}}{\partial \phi^{\star}}(-1)^{\epsilon+1} .
$$

The antifields $\phi^{\star}\left(=\frac{d \psi_{L}}{d \phi}\right)$ corresponding to the each generic field $\phi$ for this particular theory are obtained from the gauge-fixed fermion as

$$
\begin{aligned}
& B_{\mu \nu \eta}^{\star}=-\partial_{\mu} \tilde{c}_{\nu \eta}, \quad c_{\mu \nu}^{\star}=-\partial_{\mu} \tilde{\beta}_{\nu}, \quad \tilde{B}_{\mu \nu}^{\star}=\frac{1}{2} \tilde{c}_{\mu \nu}, \\
& \tilde{c}_{\mu \nu}^{\star}=\frac{1}{2} \tilde{B}_{\mu \nu}+\partial^{\eta} B_{\mu \nu \eta}, \quad \beta_{\mu}^{\star}=-\partial_{\mu} \tilde{c}_{2}, \\
& \tilde{\beta}_{\mu}^{\star}=-F_{\mu}+\partial^{\nu} c_{\nu \mu}, \quad F_{\mu \star}=-\tilde{\beta}_{\mu}, \quad \tilde{f}_{\mu}^{\star}=-\phi_{\mu}, \\
& \tilde{c}_{2}^{\star}=-\frac{1}{2} B+\partial_{\mu} \beta^{\mu}, \quad c_{1}^{\star}=\frac{1}{2} B_{2}, \quad \tilde{c}_{1}^{\star}=-\frac{1}{2} B_{1}, \\
& \phi_{\mu}^{\star}=-\tilde{f}_{\mu}, \quad B^{\star}=-\frac{1}{2} \tilde{c}_{2}, \quad B_{1}^{\star}=-\frac{1}{2} \tilde{c}_{1}, \\
& B_{2}^{\star}=\frac{1}{2} c_{1}, \quad\left\{B_{\mu \nu}^{\star}, \tilde{F}_{\mu}^{\star}, f_{\mu}^{\star}, c_{2}^{\star}\right\}=0 .
\end{aligned}
$$

Now, we construct the field-dependent parameter written in field/antifield formulation as

$$
\begin{aligned}
\Theta^{\prime} & =i \gamma \int d^{6} y\left[-B_{\mu \nu \eta}^{\star} B^{\mu \nu \eta}+\bar{B}_{\mu \nu \eta}^{\star} B^{\mu \nu \eta}\right. \\
& \left.-c_{\mu \nu}^{\star} c^{\mu \nu}+\bar{c}_{\mu \nu}^{\star} c^{\mu \nu}-\beta_{\mu}^{\star} \beta^{\mu}+\bar{\beta}_{\mu}^{\star} \beta^{\mu}\right]
\end{aligned}
$$

where bar fields are the antifields corresponding to the fields satisfying axial (noncovariant) gaugefixing condition. Under the FFBRST transformation with such field dependent parameter, the path integral measure is not invariant and give rise to a factor which is written as $e^{i S_{1}}$, and the expression for functional $S_{1}$ is calculated using Eqs. (16), (17) and (31) as

$$
\begin{aligned}
S_{1} & =\int d^{6} x\left[\kappa B_{\mu \nu \eta}^{\star}\left(\partial^{\mu} c^{\nu \eta}+\partial^{\nu} c^{\eta \mu}+\partial^{\eta} c^{\mu \nu}\right)\right. \\
& -\kappa \bar{B}_{\mu \nu \eta}^{\star}\left(\partial^{\mu} c^{\nu \eta}+\partial^{\nu} c^{\eta \mu}+\partial^{\eta} c^{\mu \nu}\right) \\
& -\kappa c_{\mu \nu}^{\star}\left(\partial^{\mu} \beta^{\nu}-\partial^{\nu} \beta^{\mu}\right)+\kappa \bar{c}_{\mu \nu}^{\star}\left(\partial^{\mu} \beta^{\nu}\right. \\
& \left.-\partial^{\nu} \beta^{\mu}\right)-\kappa \tilde{c}_{\mu \nu}^{\star} B^{\mu \nu}+\kappa \overline{\tilde{c}}_{\mu \nu}^{\star} B^{\mu \nu} \\
& +\kappa \tilde{B}_{\mu \nu}^{\star}\left(\partial^{\mu} f^{\nu}-\partial^{\nu} f^{\mu}\right)-\kappa \overline{\tilde{B}}_{\mu \nu}^{\star}\left(\partial^{\mu} f^{\nu}\right. \\
& \left.-\partial^{\nu} f^{\mu}\right)+\kappa \beta_{\mu}^{\star} \partial^{\mu} c_{2}-\kappa \bar{\beta}_{\mu}^{\star} \partial^{\mu} c_{2}+\kappa \tilde{\beta}_{\mu}^{\star} \tilde{F}^{\mu} \\
& -\kappa \overline{\tilde{\beta}}_{\mu}^{\star} \tilde{F}^{\mu}+\kappa F_{\mu}^{\star} \partial^{\mu} B-\kappa \bar{F}_{\mu}^{\star} \partial^{\mu} B \\
& -\kappa \tilde{f}_{\mu}^{\star} \partial^{\mu} B_{1}+\kappa \overline{\tilde{f}}_{\mu}^{\star} \partial^{\mu} B_{1}-\kappa \tilde{c}_{2}^{\star} B_{2} \\
& +\kappa \overline{\tilde{c}}_{2}^{\star} B_{2}-\kappa \tilde{c}_{1}^{\star} B_{1}+\kappa \overline{\tilde{c}}_{1}^{\star} B_{1}+\kappa c_{1}^{\star} B \\
& \left.-\kappa \bar{c}_{1}^{\star} B+\kappa \phi_{\mu}^{\star} f^{\mu}-\kappa \bar{\phi}_{\mu}^{\star} f^{\mu}\right] .
\end{aligned}
$$

The transformed generating functional in BV formulation is given by

$$
\begin{aligned}
Z_{\text {eff }}^{\prime} & =\int \mathcal{D} \phi e^{i\left\{W_{\Psi_{L}}+S_{1}(\kappa=1)\right\}}, \\
& \equiv \int \mathcal{D} \phi e^{i W_{\Psi_{A}}}
\end{aligned}
$$

$Z_{\text {eff }}^{\prime}$ is the generating functional for Abelian 3form gauge theory in the axial gauge with gaugefixing fermion

$$
\begin{aligned}
\Psi_{A} & =\int d^{6} x\left[-\eta_{\mu} \tilde{c}_{\nu \eta} B^{\mu \nu \eta}-\frac{1}{2} \tilde{c}_{2} B+\frac{1}{2} c_{1} B_{2}\right. \\
& -\frac{1}{2} \tilde{c}_{1} B_{1}-c^{\mu \nu} \eta_{\mu} \tilde{\beta}_{\nu}-\eta_{\mu} \tilde{c}_{2} \beta^{\mu}+\frac{1}{2} \tilde{c}_{\mu \nu} \tilde{B}^{\mu \nu} \\
& \left.-F^{\mu} \tilde{\beta}_{\mu}-\tilde{f}^{\mu} \phi_{\mu}\right] .
\end{aligned}
$$

The extended action $W_{\Psi_{A}}$ for 3-form gauge theory in axial gauge also satisfies the quantum master equation (29). This result is also true for the connection of generating functionals in any covariant and noncovariant gauges in $\mathrm{BV}$ formulation.

\section{CONCLUSIONS}

The 3-form gauge theory in noncovariant gauge has been developed with the help of the finite field 
dependent BRST transformation. Usual BRST transformations have been generalized to the case of Abelian 3-form gauge theory in covariant gauge. The generating functional for this theory is shown to be connected to that of in the noncovariant (axial) gauge through FFBRST transformation. We established this connection by constructing appropriate finite field dependent BRST parameter. However, the various noncovariant gauges like the Coulomb gauge, the light-cone gauge, the planer gauge and the temporal gauge can also be studied under such formulation. Thus, our formulation enables to study the 3-form gauge theory in noncovariant gauges by this connection. We further extend these results in the context of BV formula- tion. It will be interesting to see how the different Green functions are related in different gauges for this theory. Further analysis of FFBRST transformation of quantum gravity, BLG theory, ABJM theory and higher derivative field theory will be subject of interest.

\section{Acknowledgments}

SU gratefully acknowledges the financial support from the Council of Scientific and Industrial Research (CSIR), India, under the SRF scheme.
[1] George Leibbrandt, Rev. Mod. Phys. 59, 1067 (1987).

[2] George Leibbrandt, Noncovariant Gauges: Quantization of Yang-Mills and Chern-Simons Theory in Axial-Type Gauges, (World Scientific, 1994).

[3] V. N. Gribov, Nucl. Phys. B 139, 1 (1978).

[4] A. P. Szczepaniak and E. S. Swanson, Phys. Rev. D 65, 025012 (2001).

[5] R. R. Metsaev, C. B. Thorn and A. A. Tseytlin, Nucl. Phys. B 596, 151 (2001).

[6] L. Brink, O. Lindgren and B. E. W. Nilsson, Phys. Lett. B 123, 323 (1983).

[7] A. Gustavsson, JHEP 1201, 057 (2012).

[8] H.-C. Kim, S. Kim, E. Koh, K. Lee, S. Lee, JHEP 12,031 (2011).

[9] C.-S. Chu and D. J. Smith, JHEP. 0904, 097 (2009).

[10] J. Bagger and N. Lambert, JHEP. 0802, 105 (2008).

[11] J. Bagger and N. Lambert, Phys. Rev. D 77, 065008 (2008).

[12] A. Gustavsson, JHEP 0804, 083 (2008).

[13] M. Faizal, JHEP 1204, 017 (2012).

[14] M. Faizal, B. P. mandal and S. Upadhyay, arXiv:1212.5653 [hep-th].

[15] P.-M. Ho, Chin. J. Phys. 48, 1 (2010)

[16] M. B. Green, J. H. Schwarz and E. Witten, Superstring Theory Vols 1 and 2 (Cambridge University Press: Cambridge, 1987).

[17] J. Polchinski, String Theory Vols 1 and 2 (Cambridge University Press: Cambridge, 1998).

[18] D. Lust, S. Theisen, Lectures in String Theory
(Springer-Verlag: New York, 1989).

[19] S. Upadhyay and B. P. Mandal, Eur. Phys. J. C 72, 2059 (2012).

[20] S. D. Joglekar and B. P. Mandal, Phys. Rev. D 51, 1919 (1995).

[21] R. Banerjee and B. P. Mandal, Phys. Lett. B 27, 488 (2000).

[22] S. D. Joglekar and B. P. Mandal, Int.J. Mod.Phys. A 17, 1279 (2002).

[23] S. Upadhyay and B. P. Mandal, Eur. Phys. Lett. 93, 31001 (2011).

[24] S. Upadhyay, S. K. Rai and B. P. Mandal, J. Math. Phys. 52, (2011) 022301.

[25] S. Upadhyay and B. P. Mandal, Eur. Phys. Lett. 93, 31001 (2011); Mod. Phys. Lett. A 40, 3347 (2010) .

[26] B. P. Mandal, S. K. Rai and S. Upadhyay, Eur. Phys. Lett. 92, 21001 (2010).

[27] S. Upadhyay and B. P. Mandal, Eur. Phys. J. C 72, 2065 (2012).

[28] S. Upadhyay and B. P. Mandal, Annals of Physics 327 2885(2012).

[29] S. Upadhyay and B. P. Mandal, AIP Conf. Proc. 1444, 213 (2012).

[30] M. Henneaux and C. Teitelboim, Quantization of gauge systems (Princeton, USA: Univ. Press, 1992).

[31] S. Weinberg, The quantum theory of fields, Vol-II: Modern applications (Cambridge, UK Univ. Press, 1996). 